\title{
Erratum to: Early diagnosis of pancreatic necrosis based on perfusion CT to predict the severity of acute pancreatitis
}

\author{
Yoshihisa Tsuji ${ }^{1,15,16} \cdot$ Naoki Takahashi $^{2} \cdot$ Hiroyoshi Isoda $^{3} \cdot$ Koji Koizumi $^{4}$. \\ Sho Koyasu ${ }^{3}$ - Miho Sekimoto ${ }^{5}$ - Yuichi Imanaka ${ }^{5}$ - Shujiro Yazumi ${ }^{6}$ • \\ Masanori Asada $^{6} \cdot$ Yoshihiro Nishikawa $^{6} \cdot$ Hiroshi Yamamoto $^{7} \cdot$ Osamu Kikuchi $^{7}$. \\ Tsukasa Yoshida $^{7}$ - Tetsuro Inokuma ${ }^{8}$. Shinji Katsushima ${ }^{9} \cdot$ Naoki Esaka $^{9}$. \\ Akihiro Okano $^{10}$ - Chiharu Kawanami ${ }^{11}$ - Nobuyuki Kakiuchi ${ }^{11} \cdot$ Masahiro Shiokawa $^{11}$. \\ Yuzo Kodama $^{1}$ - Ichiro Moriyama ${ }^{12}$. Takafumi Kajitani $^{13}$ - Yoshikazu Kinoshita ${ }^{14}$. \\ Tsutomu Chiba ${ }^{1}$ \\ Published online: 26 April 2017 \\ (c) Japanese Society of Gastroenterology 2017
}

\section{Erratum to: J Gastroenterol \\ DOI 10.1007/s00535-017-1330-5}

In the original publication of the article, the author names were swapped in the author group. The corrected list of author names is presented above.

The original article was corrected.

The online version of the original article can be found under doi:10.1007/s00535-017-1330-5.

Yoshihisa Tsuji

ytsuji@kuhp.kyoto-u.ac.jp;

ytsuji@belle.shiga-med.ac.jp

1 Department of Gastroenterology and Hepatology, Kyoto University Hospital, Kawahara-cho, Shogoin, Sakyo-ku, Kyoto 606-8507, Japan

2 Department of Radiology, Mayo Clinic, 200 1st St SW, Rochester, MN 55902, USA

3 Department of Radiology, Kyoto University Hospital, Kawahara-cho, Shogoin, Sakyo-ku, Kyoto 606-8507, Japan

4 Division of Clinical Radiology Service, Kyoto University Hospital, Kawahara-cho, Shogoin, Sakyo-ku, Kyoto 606-8507, Japan

5 Department of Healthcare Economics and Quality Management, Kyoto University Hospital, Kawahara-cho, Shogoin, Sakyo-ku, Kyoto 606-8507, Japan

6 Digestive Disease Center, Kitano Hospital, The Tazuke Kofukai Medical Research Institute, 2-4-20 Ohgimachi, Kitaku, Osaka 530-8480, Japan
7 Department of Gastroenterology and Hepatology, 1 Chome1-1 Miwa, Kurashiki, Okayama 710-0052, Japan

8 Department of Gastroenterology and Hepatology, Kobe City Medical Center General Hospital, 2-2-1 Minatojima-minamimachi, Chuo-ku, Kobe, Hyogo 650-0047, Japan

9 Department of Gastroenterology and Hepatology, Kyoto Medical Center, 1-1 Fukakusa, Mukaihata-cho, Fushimi-ku, Kyoto 612-8555, Japan

10 Department of Gastroenterology and Hepatology, Tenri Hospital, 200 Mishima-cho, Tenri, Nara 632-8552, Japan

11 Department of Gastroenterology and Hepatology, Japanese Red Cross Otsu Hospital, 1-1-35 Nagara, Otsu, Shiga 520-8511, Japan

12 Division of Clinical Study of Oncology, Shimane University School of Medicine, 1060 Nishikawatsucho, Matsue, Shimane 690-8504, Japan

13 Department of Radiology, Shimane University School of Medicine, 1060 Nishikawatsucho, Matsue, Shimane 690-8504, Japan 
14 Department of Gastroenterology and Hepatology, Shimane University School of Medicine, 1060 Nishikawatsucho, Matsue, Shimane 690-8504, Japan

15 Department of Gastroenterology and Hepatology, The Japan Baptist Hospital, 47 Kitashirakawa Yamanomotocho, Sakyoku, Kyoto 606-8273, Japan

16 Present Address: Shiga University of Medical Science, SetaTsukinowacho, Otsu, Shiga 520-2121, Japan 